\title{
Variations
}

Variations

Revue internationale de théorie critique

18 | 2013

"La poésie est une arme chargée de futur »

\section{Le grand bond en avant de la charité}

Eclairages cantonais

Monique Selim

\section{(2) OpenEdition}

Journals

Édition électronique

URL : http://journals.openedition.org/variations/634

DOI : $10.4000 /$ variations. 634

ISSN : 1968-3960

Éditeur

Les amis de Variations

Référence électronique

Monique Selim, « Le grand bond en avant de la charité », Variations [En ligne], 18 | 2013, mis en ligne le 31 mai 2013, consulté le 20 avril 2019. URL : http://journals.openedition.org/variations/634 ; DOI :

10.4000/variations.634

Ce document a été généré automatiquement le 20 avril 2019

Les ami•e•s de Variations 


\title{
Le grand bond en avant de la charité
} Eclairages cantonais

\author{
Monique Selim
}

1 Dès les années 80 , l'expression charity business fait son apparition pour désigner l'ampleur des flux monétaires qui sont impliqués dans les actions caritatives. À cette époque - qui reste marquée par la partition du monde entre pays communistes et capitalistes - ne sont cependant concernées que les démocraties d'ancienne industrialisation qui ciblent dans des sociétés lointaines leurs victimes bénéficiaires. Les logiques sont univoques et s'inscrivent dans les catégories idéologiques de l'Occident et du/des Suds. Au sein d'une société comme la France, la charité en destination de ceux qu'on appelle pudiquement par différents vocables tous politiquement connotés - les plus «démunis", «défavorisés", etc. - reste peu visible, largement coutumière et rituellement d'inspiration confessionnelle. Le "pauvre ", comme figure symbolique s'incarnant dans des cohortes d'individus peuplant les rues et les parcs des villes où ils bâtissent leurs abris de fortune, n'a pas encore fait sa percée médiatique. À l'aube du XXI siècle le paysage a radicalement changé, non seulement ici, mais ailleurs. Avec la crise globale du capitalisme financiarisé, la pauvreté s'est répandue dans des sociétés qui ont basculé vers la désindustrialisation, frappées par les fermetures d'usine, les licenciements et le chômage. Ainsi la charité fait un retour triomphal sous des visages multiples dans la conjoncture française, exemplaire des processus en cours : ceux qu'on y dénomme les bénévoles se multiplient et en viennent à se trouver beaucoup plus nombreux que les travailleurs sociaux professionnels débordés au chevet des sans domicile fixe - SDF expression qui, à elle seule, résume les déplacements, expulsions, migrations qui sont leur lot quotidien. Au-delà du fait qu'une partie de ces bénévoles affiche une couleur religieuse (catholicisme, protestantisme, islam, etc.), cette mobilisation se présente comme un symptôme social diffus de sentiments contradictoires: la peur qui hante chacun de se retrouver dans la même situation critique, l'urgence de contrecarrer cette identification létale, l'angoisse de voir l'ordre social se disloquer sous l'effet d'une misère croissante qui s'infiltre partout, l'idée majeure enfin que l'Etat s'est déjà retiré de la problématique de la paupérisation et qu'il faut gérer à sa place. Dans le même moment la 
figure du pauvre s'est psychiatrisée : si la pauvreté était déjà assimilée à une pathologie sociale, le pauvre y a désormais ajouté la variable d'une déficience mentale explicative. $\mathrm{Au}$ « clochard » des années 50, au « marginal » des années 60-70, contre le « système », au chômeur des années 80-90, s'est désormais substituée l'image du pauvre « fou », bipolaire, psychotique et/ou schizophrène. Hors de cette configuration paradigmatique, si l'on tourne le regard vers d'autres régions éloignées, on perçoit, sous des modalités diverses, combien la charité a envahi le monde global et est actuellement érigée en réponse à des inégalités économiques grandissantes et à un abandon des politiques de redistribution. Les ONG, entreprises transnationales dotées d'agences de street fundraising, ont pris une place décisive dans l'extension et la généralisation de l'hypothèse charitable au point que celle-ci a acquis une position hégémonique, dépassant les frontières des systèmes politiques. En effet dans un contexte où la violence des forces du marché partout présentes cherche son exorcisme idéel dans un paquet globalisé de normes ethicomorales, la charité resplendit par sa moralité intrinsèque et son visage solidaire.

\section{La promotion politique du volontariat à Canton}

2 Deuxième puissance économique mondiale, la Chine - dont l'Etat-parti communiste avec ses capacités de coercition, a pleinement favorisé le développement capitaliste témoigne avec acuité du rayonnement actuel de la charité et c'est sur les facettes singulières de son déploiement que nous nous tournerons maintenant nous fondant sur les investigations anthropologiques menées en 2007. Dirigeons-nous vers Canton, capitale de la province du Sud, premier moteur de croissance et modèle actuel de gestion politique « ouverte " pour le gouvernement issu du XVIII ${ }^{e}$ congrès du parti communiste chinois de 2012. Deux organisations de volontaires y mettent en scène un activisme bénévole remarquable. La première organisation - zhi yuan zhe - est créée en 1995 par la ligue de la jeunesse communiste, sous tutelle du Parti. La seconde organisation - yi gong lian ( guangzhou vonlonteer union) - est inscrite en 2002 au bureau des affaires civiles de la municipalité. Les deux organisations, loin d'être en rivalité, fonctionnent comme des stimulants mutuels et l'on observe que les individus sont souvent inscrits dans les deux, oubliant la spécificité de chacune dans l'enthousiasme des actions. Comme dans tous les champs sociaux, Internet joue dans le volontariat un rôle considérable car c'est sur la Toile qu'on s'inscrit dans ces organisations qui disposent de sites riches et attractifs. Les revendications émergent ici comme ailleurs que les volontaires soient défrayés de tous leurs frais de déplacement, de repas et de boissons durant leur temps de volontariat. Celui-ci est en effet de plus en plus appréhendé comme un travail et les petits carnets dont les acteurs disposent et où sont notées les heures de volontariat qu'ils accomplissent viennent accréditer cette perception encore renforcée par leur badge qui conforte leur appartenance.

2008 est pour le volontariat chinois une année exceptionnelle qui intensifie propagande et initiative en raison de deux événements phares; les Jeux Olympiques à Pékin où les volontaires font l'objet d'une sélection drastique et le tremblement de terre du Sichuan où accourent spontanément des milliers de jeunes, ensuite refoulés par les autorités qui craignent des débordements, car la corruption s'est révélée une cause déterminante de l'écroulement sur les enfants des «écoles en miettes de soja ». À Canton une tempête de neige inédite constitue une troisième occurrence qui voit se précipiter à la gare toutes les bonnes volontés. Outre ces épisodes, le volontariat s'oriente dans deux grandes directions 
sur lesquelles nous nous pencherons. En premier lieu tous ceux qui rentrent en milieu urbain dans l'ensemble que forment les "faibles" - ruo shi qunti, c'est-à-dire les "pauvres" - où se remarquent les personnes âgées, à leur domicile ou en maison de retraite qui font l'objet de tous les soins, mais aussi les handicapés de tous âges, physiques, mentaux, autistes, etc. L'éducation rurale est un second champ essentiel du volontariat cantonnais qui cible les élèves dont les parents sont migrants : minorités nationales (Yao, etc.), villages de montagne d'accès difficile sont alors l'occasion de séjours courts durant les vacances des jeunes urbains qui découvrent là d'autres conditions de vie et un étranger de l'intérieur qu'ils ignorent et qui concrétise une altérité radicale. La première organisation de volontaires en milieu rural naît en 2001 à Canton, et 10 ans après un congrès en réunit 200 sur les 500 qui existeraient actuellement dans toute la Chine. Le développement de ce type de volontariat s'intègre en effet dans un mouvement plus vaste qui voit grandir les préoccupations environnementales et l'attirance pour la nature et débouche sur le tourisme rural, l'achat direct et contractuel auprès de paysans de leur récolte, des échanges organisés entre familles urbaines et rurales, etc.

4 Jeunes et plus âgés, hommes et femmes, du Parti ou non, travailleurs, étudiants, licenciés - xiagang - ou chômeurs, tous les profils se donnent à voir parmi les volontaires. Dans le contexte d'un régime autoritaire comme la Chine, le volontariat est une hydre à plusieurs têtes. L'intérêt de l'Etat-parti dans le lancement du volontariat ressortit à l'encadrement, la surveillance et le contrôle de larges parties de la population oscillant entre l'écœurement devant la corruption généralisée régnante et la compassion face aux plus démunis et aux exclus de la course à la consommation. Internet, en ouvrant les portes vers le monde global et en donnant aux acteurs la capacité de former des groupes d'action et de concrétiser collectivement leurs souhaits, a eu l'effet d'un détonateur qui a appelé, de la part du gouvernement, la constitution d'organes de canalisation à la fois idéologique et pratique : les organisations de volontaires sont de telles machines, aptes à orienter et discipliner les désirs, à freiner les influences des institutions étrangères promptes à financer toute action tendanciellement contestataire, à maintenir les agencements collectifs dans un cadre politique conforme. La nécessité en apparait d'autant plus urgente à Canton, que Hong Kong est mitoyen et que des ONG non enregistrées localement se multiplient en résonance aux ONG étrangères avec lesquelles elles tissent des liens numériques et personnalisés et dont elles se réapproprient les modèles d'action. Les organisations de volontaires tentent donc de ramener dans le giron de l'Etat toutes ces énergies qui pourraient dériver vers une "société civile» libre et s'efforce de s'autoinstituer en matrice principielle d'une « société civile » pacifiée et « harmonieuse ». Les formations de volontaires sont par conséquent très nombreuses. Il ne fait donc aucun doute que les organisations de volontaires s'érigent en appareil idéologique d'Etat, et ce d'autant plus que les autorités établissent une continuité imaginaire entre Lei Feng, le héros de la période maoïste mort électrocuté très jeune et les volontaires actuels, continuité qui valorise les plus âgés et légitime toutes les générations. Par ailleurs, si les organisations de volontaires sont dirigées par des cadres politiques de confiance qui installent et font respecter un fonctionnement hiérarchique, cependant une relative souplesse règne en regard des organisations de masse (femmes, jeunes, syndicats) traditionnelles, donnant l'illusion d'une plus grande indépendance, en particulier aux jeunes et aux étudiants, qui, rappelons le, peuvent capitaliser leur volontariat en points comptant dans leurs examens. 
Venant d'horizons très divers, se mêlant dans les actions sur un plan d'égalité, les acteurs trouvent dans le volontariat, en Chine comme ailleurs, un mode de socialisation débouchant éventuellement sur des réseaux de sociabilité qui peuvent s'autonomiser et perdurer. Mais, compte tenu de la verticalité qui continue à présider aux ordonnancements sociaux du fait du monopole de l'autorité politique, la fonction de passerelle entre les classes sociales, que revêt partout le volontariat, prend ici une connotation singulière. L'extraordinaire et fulgurante augmentation des écarts de revenus depuis maintenant plus de trois décennies, l'hégémonisation de la consommation et l'ostentation systématique des richesses ont d'autant plus accru les perceptions négatives de l'inégalité que l'égalité fut antérieurement célébrée à l'époque maoïste et fait trace dans les mémoires. Que les inégalités soient le fruit d'une hiérarchisation politico-économique, qui engrange le capital économique sur le capital politique, apparaît de plus en plus flagrant aux yeux de tous, les scandales se multipliant en haut de la pyramide. Dans cette configuration, le volontariat émerge comme une sorte de réparation symbolique de l'inégalité, par la relation personnalisée qu'il inaugure entre un individu quelle que soit sa position sociale - et plus «malheureux » que lui-même, en état par conséquent d'infériorité et de vulnérabilité. Si donc l'exercice de capture idéologique et politique que met en marche le volontariat se révèle efficace, c'est en raison de l'emprise qu'il tisse sur les acteurs, leur donnant le sentiment qu'il laisse place à leurs subjectivités plurielles. Tout se passe comme si, dans l'imaginaire, la société était transformée en un univers où chacun devient volontaire face à plus faible que soi et intègre tout arrivant comme un potentiel volontaire, ce qui d'ailleurs est le cas révélateur avec l'anthropologue. Une réciprocité abyssale prend forme faisant du corps social le champ d'une entraide généralisée, animée par l'hypostase de la charité, sublimant tout à la fois rapports sociaux et idiosyncrasies confondues. L'écoute des sujets, cherchant à expliquer leur devenir volontaire ouvre ainsi sur la coagulation de plusieurs logiques ; parmi celles$c i$, certaines font appel à un calcul de type investissement à long terme : le volontariat au présent serait dans l'avenir récompensé par un retour lorsque le sujet en aurait besoin, "ça me sera rendu» dit-on. On distingue aussi la dimension psychique de l'investissement émotionnel: " plaisir », "calme», " apaisement » produit par l'acte volontaire; tous ces mots énoncés dressent les contours d'une jouissance retirée de l'altruisme, puisque « aider les autres c'est s'aider soi-même ». La charité apparaît alors comme une saisie de l'Autre pour se retrouver/s'oublier, postures apparemment opposées mais qui fusionnent dans l'inconscient des sujets lorsqu'ils évoquent «ça ». Dispositif sociopolitique d'implication subjective, le volontariat faciliterait la fabrique d'une armée de réserve immédiatement mobilisable lors d'événements tragiques, en raison du psychopouvoir qu'il recèlerait. La distribution de prix aux volontaires, l'héroïsation des meilleurs sur le même mode qu'au Parti ou dans une organisation de masse, en font en effet un bien commun idéal. Il s'agit de "prendre des responsabilités sociales, faire quelque chose pour la société » puisque l'Etat chinois ne pourrait prendre en charge les multitudes de pauvres qui existent. Dans un contexte où le travail social n'a fait son apparition que dans les années 90 et où peu à peu il se professionnalise mais n'est pas encore totalement enraciné dans les mentalités, le volontariat entretient des liens ambigus avec le travail social, entre concurrence et confusion, tout en maintenant des fils généalogiques avec le militantisme de type politique. Dans le même moment il participe à l'institutionnalisation d'une société de services, au sein de laquelle se conjuguent les services charitables et les services marchands, ces derniers relevant des équipes de travailleurs sociaux contractualisées à l'année et financées depuis 2012 après réponses 
aux appels d'offre gouvernementaux. Marchandises ou dons, ces services nourrissent le paradigme du care, du souci des autres postulé comme fondement de l'ordre social.

\section{Investissements charitables en ville}

En allant à la rencontre de quelques volontaires représentatifs des différents groupes en jeu, le lecteur percevra mieux combien le volontariat à Canton active et englobe des lignes divergentes, qui, tirées vers leurs propres finalités, conduisent autant aux manipulations politico-idéologiques de l'Etat-parti qu'aux désirs d'émancipation et de transformation des acteurs, au contact des trous noirs de la société. Pour éviter une interprétation trop normative $\mathrm{du}$ volontariat, commençons par quelques voix de volontaires manifestant un recul certain face aux canevas dans lesquels ils sont pris. Fils d'une enseignante et d'un ouvrier qui habitent Canton, Aric est un jeune homme de 25 ans, qui, après avoir raté le concours de l'université puis suivi trois années d'études techniques, s'est engagé dans la vie professionnelle et vit seul. Devenu employé de bureau dans une entreprise qui ne lui accorde aucun jour de congé, Aric se lance dans le volontariat en 2005 pour élargir son horizon, étendre ses relations, rompre l'ennui profond qu'il ressent. L'entreprise le licencie lorsqu'elle trouve qu'il consacre trop de temps à ses activités bénévoles et Aric, qui a fait quelques économies, est au chômage depuis un an et profite de sa nouvelle disponibilité pour intensifier son volontariat. Il a une première expérience de volontariat auprès d'une personne âgée à qui il s'attache. Sa mort le conduit à se détourner des «fins de vie » et il rejoint alors l'organisation de volontaires sous la tutelle de la ligue de la jeunesse (zhi yuan zhe) qui l'envoie au cœur du vieux quartier de Canton dans un centre modèle. Très vite il conteste les directives de son chef d'équipe, considérant que son désir de volontariat est instrumentalisé dans une perspective de propagande, "qu'il ne s'agit que de publicité». Les rapports avec cette organisation sont rompus et il se tourne vers la seconde organisation de volontaires ( $y i$ gong lian) sous la tutelle du bureau des affaires civiles de la municipalité, qu'il estime beaucoup plus souple et qui lui permet de rester dans le centre social du vieux quartier où il surveille deux soirs par semaine des enfants dans une salle d'étude et de divertissement. Il est investi aussi dans un projet très récent (2008) qu'il considère comme une véritable ONG « indépendante » dit-il et qui est financé par un Américain d'origine chinoise : il s'agit de bâtir une "relation de confiance » avec un enfant villageois laissé seul par ses parents paysans migrants partis travailler en ville, relation épistolaire mais aussi concrète puisque le volontaire doit rendre visite une fois par an à l'enfant. Dénommé "grand ami/petit ami », ce projet a été découvert par Aric sur la Toile où il a dû remplir un formulaire qui lui a paru très compliqué et qui demandait beaucoup de patience. Il a été ensuite convoqué au bureau de l'ONG, sélectionné et a reçu une formation pour établir et entretenir la relation avec l'enfant. Il a choisi sur photo « son enfant ", comme 300 autres jeunes volontaires. Outre son activité au centre social et cette relation privilégiée avec le petit villageois, Aric rend visite à des orphelins au Nord de Canton et ce bénévolat diversifié ponctue son temps. Très sérieux, doté d'un solide esprit critique, Aric a nettement refusé que sa bonne volonté soit l'objet d'un embrigadement de caractère politique et il a pris délibérément la direction opposée, celle des ONG et d'une « société civile » idéalisée.

7 Il n'est pas le seul, comme l'indique un autre jeune homme, Dorgon, qui affiche un scepticisme appuyé et tient à montrer qu'il n'est pas dupe de la « machinerie volontaire ». 
Fils de paysans, Dorgon vient de terminer ses études de chimie à l'université dont, faute de bonnes notes, il n'est ressorti qu'avec un diplôme d'informatique spécialisée en pharmacie. Il a été embauché à un salaire très bas par une compagnie d'assurance au sein de laquelle plusieurs de ses collègues, que nous retrouverons bientôt, tentent de le convaincre de se joindre à elles dans leurs activités de volontaires. Mais Dorgon résiste fermement, persuadé qu'en Chine le volontariat est toujours téléguidé par le gouvernement et que «les gens ne sont pas sincères ». Une longue tirade, au cours de laquelle il relate son expérience, vise à le prouver :

depuis l'âge de 10 ans, j'ai participé au groupe de volontaires de l'école, mais bien sûr ce n'était pas totalement volontaire. C'est toujours les professeurs qui commandent en disant "on va apprendre de Lei Feng » et tout le monde sortait avec les balais et les serpillières... et à l'université c'était pareil, c'était la ligue de la jeunesse qui nous commandait d'être volontaire. Mais Lei Feng on ne comprenait rien! Au collège on voulait simplement que notre classe gagne car toutes les classes étaient en concurrence et c'était un honneur de gagner. Lei Feng on s'en fichait. À l'université aussi c'était l'émulation collective mais jamais «volontairement». Le volontariat c'est jamais sans compensation. Chacun trouve quelque chose selon ses objectifs. Moi quand j'ai fait 24 jours de volontariat pendant les vacances d'été où je devais faire le ménage dans l'école, c'est parce que, alors je pouvais regarder les Jeux Olympiques.

8 Cette narration, centrée sur l'enrôlement et l'endoctrinement systématiques qui président toujours au modèle éducatif chinois, met en évidence les limites des conceptions autoritaires du volontariat et d'un mode d'inculcation en décalage avec les aspirations d'une partie des jeunes.

En revanche, dès lors que des jeunes repèrent une voie de professionnalisation dans un volontariat qui est alors très élitiste - comme lors des Jeux Olympiques de Pékin -, ils se préparent à y concourir, ce qui suppose néanmoins d'en avoir les moyens matériels et l'assurance personnelle. Etre sélectionné pour devenir volontaire aux Jeux Olympiques de Pékin est ainsi le fruit d'une procédure longue et stricte qui retint seulement mille jeunes sur 2000 postulants dans le Guangdong. Recrutés dans les universités par la ligue de la jeunesse - sous tutelle au Parti - les candidats devaient s'être déclarés dès 2006. Les 100 gagnants ont reçu une formation de 3 jours dans un grand hôtel. Parmi ceux-ci, 10 venaient d'entreprises ayant versé des financements pour les Jeux Olympiques. Quant aux autres, ils ont dû déboursé entre 4000 et 7000 yuans $^{1} .7000$ yuans, telle est la somme qu'à payée la mère de Xia He, cadre dans une entreprise, divorcée et élevant seule sa fille. Xia He a remarqué que sur les 100 volontaires de la province partis à Pékin, à ses yeux au moins 20 viennent de familles "riches ", alors qu'elle se classe elle-même dans la couche « moyenne ». Le père d'un volontaire était - dit-elle - connu comme arbitre international. «L'honneur ", la « fierté personnelle » le sentiment de contribuer à la gloire de la nation, et en même temps d'élargir ses visions du monde animent Xia He qui exhibe avec joie les "souvenirs» de Pékin : un certificat, une carte d'identité des JO, des dossiers, un livre reçu pour la formation, son carnet de notes, etc. «Durant 30 jours on était comme à la guerre, tous des camarades fusionnels, solidaires » ajoute la jeune fille encore émue, en se remémorant cette expérience gratifiante. C'est avec mépris, en revanche, qu'elle évoque les activités usuelles de volontaires auprès des handicapés ou des personnes âgées, avouant sans vergogne son manque d'intérêt. Les projets de Xia He sont clairs: partir étudier à l'étranger, de préférence en Espagne, quitter Canton, la Chine, sa mère dont elle pense recevoir 300000 yuans pour démarrer sa nouvelle aventure, très éloignée des idées de charité et de compassion généralement associées au volontariat! Pour Xia He, comme 
pour deux de ses camarades cantonaises rencontrées à Pékin, être consacré volontaire dans de telles circonstances est un tremplin pour l'avenir. Rappelons que dans des questionnaires d'embauche, le volontariat marque éventuellement des points, comme pour les étudiants.

Tournons-nous vers d'autres générations de femmes dans l'espace social du volontariat cantonais; elles mettent en lumière des processus bien repérés ailleurs de transfert d'investissement et de déplacement d'occupation, mais dans une version spécifique liée au contexte politico-économique chinois. Lin Yao, 50 ans, en est un exemple frappant: ancienne cadre d'un restaurant d'Etat qui fait faillite à la fin des années 90, elle est licenciée (xiakang) en 95 alors même qu'elle a obtenu son intégration au Parti en 1994. Sa loyauté au Parti est, comme c'est souvent le cas, une forme de dépassement des épreuves que le Parti a fait subir à sa famille. Les grands-parents paternels sont issus de Dongquan et partent à Hong Kong au moment de la guerre sinojaponaise. Le père de Lin Yao rentre à Canton après la Libération, devient fonctionnaire, épouse une infirmière et tous deux se dévouent au Parti. Lors de la famine du Grand Bond en avant, la mère de Lin Yao fait appel à sa belle-famille restée à Hong Kong, reçoit des boites de lait en poudre et en donne à quelques-unes de ses collègues. Elle est alors accusée durant la révolution culturelle d'avoir vendu du lait de Hong Kong et son mari est envoyé en rééducation à la campagne, malgré les félicitations du Parti qu'il avait reçues après une vasectomie volontaire, au troisième enfant. En dépit de tout, le Parti reste l'horizon identitaire de la famille et Lin Yao ne déroge pas à cet idéal, bien dur à soutenir - dit-elle en riant - dans la période actuelle où la corruption règne dans l'appareil d'autorité faisant tomber la réputation des membres du Parti. Au chômage depuis 10 ans, Lin Yao a renoncé à chercher du travail, consciente que son âge était un obstacle rédhibitoire. L'apparition de l'organisation de volontaires sous la tutelle de la ligue de la jeunesse la ravit en 1995 (zhi yuan zhe), et, face au temps libre dont elle dispose après son licenciement, elle saute le pas et s'investit à fond dans ce nouveau champ qui lui permet une continuité avec son militantisme au Parti. Elle se concentre tout d'abord sur les personnes âgées en maison de retraite, auxquelles elle rend visite puis créée en 2006 sa propre équipe de volontaires qui se consacre aux enfants en milieu rural. C'est en mettant une annonce dans le grand quotidien de la province du Guangdong et en lançant un groupe sur $\mathrm{qq}^{2}$ qu'elle rassemble une centaine de personnes. Son équipe prend place dans l'organigramme hiérarchique de zhi yuan zhe: au-dessus d'elle, elle un responsable qui est manager à China Télécom, comme, dit-elle, tous les "supérieurs» sont des "gens haut placés, professeurs, médecins, etc.» qui sont nommés. Elle dirige son équipe qui comprend 10 leaders avec l'aide de l'organisation qu'elle juge très professionnelle et en collaboration avec les bureaux locaux chargés de l'éducation et la fédération des femmes sous tutelle du Parti. L'équipe part en «mission » dans les villages les plus pauvres de la région pour donner aux enfants livres, stylos, papiers offerts par des entreprises qui souvent délèguent aussi des groupes de leurs salariés dans ces croisades humanitaires vers l'Autre intérieur. Lin Yao avoue avoir été très traumatisée par son chômage prolongé et s'être reprise avec ce bénévolat; elle est particulièrement fière de «son équipe " qui a une petite carte d'identité spécifique où on la voit en photographie avec une personne âgée, les deux femmes proches, souriantes, heureuses. Sur un autre document de l'équipe une bougie totalise le nombre d'heures bénévoles qu'elle a personnellement effectuées : $600 \mathrm{~h}$, le maximum, insiste-t-elle, compte tenu des « étages » de la bougie qui montent par palier de 50 ! En regard de cette activité sociopolitique de volontariat qui rehausse son image d'elle-même, Lin Yao semble accorder une moindre importance à sa fille unique qui 
travaille dans une banque et à son mari, salarié dans un restaurant. L'intégration dans l'organisation des volontaires est ici devenue un travail, de surcroit politique au sens plein du terme.

11 Participer à une "soupe d'amour " pour les sinistrés du Sichuan, téléphoner à 10 personnes âgées 5 minutes chacune pour les distraire, emmener son fils de 10 ans rendre visite régulièrement à une femme pauvre isolée pour lui apprendre à bien se conduire, réparer gratuitement des objets dans un "marché de volontaires » édifié sous forme de kiosque où les habitants du quartier vont apporter des ustensiles en panne, monter des spectacles édifiants, s'occuper d'enfants dits «spéciaux» (autistes), la panoplie est immense et il suffit de cliquer sur la case choisie sur Internet. Tous ces actes unifient les sujets dans l'idée d'une charité vivace et vivante, susceptible aussi d'effacer la culpabilité. Dans cette version racheter ses fautes antérieures, verser une sorte de compensation pratico-symbolique n'est pas loin des mérites bouddhistes et l'on comprend aisément que l'on retrouve dans la multitude des volontaires des convertis récents ou plus anciens au bouddhisme, à la suite d'un drame personnel qui les a conduits à rencontrer un "Maître ", à suivre son enseignement et a devenir bénévole dans un temple en même temps que dans l'une des deux organisations officielles. Mais cette conception déculpabilisante de la charité est aussi prônée par des militants politiques du volontariat telle cette ancienne directrice d'école - fille de dignitaire du Parti - qui a rejoint yi gong lian dès sa création et qui entonne avec une joie éclatante les chants de Lei Feng. Responsable d'un service dans la fédération des femmes, cette femme désormais à la retraite, égrène la liste des innombrables prix qu'elle a reçus pour ses volontariats tous azimuts dans des centres de détention, auprès de jeunes à qui elle a donné des conseils psychologiques, sans oublier les mendiants qu'elle se serait chargée - selon un bel euphémisme - de convaincre de rentrer dans leur province natale: prix du meilleur volontaire de Canton (réservé à une élite de 10), prix du volontaire d'or de la province, "Ambassadrice de la civilisation à Canton », etc.

12 Attirons l'attention sur cette fonction inattendue que réalise le volontariat entre adeptes d'une morale bouddhique - dont la subversion potentielle est dès lors neutralisée - et cadres politiques œuvrant dans un esprit léniniste caractérisé par des schèmes hiérarchiques inexpugnables. Ainsi notre ténor féminin du volontariat souligne « qu'on se sent mieux lorsqu'on donne que lorsqu'on reçoit » et que «donner, aider, procure une satisfaction spirituelle». Elévation morale, économique, politique nourrissent ainsi le comportement charitable du volontaire actuel.

13 Evincer la culpabilité - celle par exemple de ne pouvoir prendre en charge sa mère âgée faute de ressources et de place dans le cas d'une jeune femme issue d'une famille ouvrière - mais aussi annihiler la réflexion car « réfléchir mène à la tristesse en Chine tant il y a de problèmes » - nous dit une jeune comptable appréciant peu son emploi - telles seraient les vertus de la charité volontaire, potion étourdissante et enthousiasmante à la fois, nécessaire pour supporter une quotidienneté ingrate, frustrante dans une conjoncture de concurrence effrénée.

\section{Vers les campagnes}

14 Le volontariat de jeunes urbains en milieu rural constitue dans la nouvelle praxis de la charité un paradigme notable dans la mesure où il s'adresse à un autre inconnu, surface de projections polychromes impliquant apprentissages et précautions face à cette sorte 
de «naturel » que serait le «paysan». On en prendra pour exemple une organisation pionnière dans ce domaine de l'éducation en milieu rural qu'on dénommera Dzeta. Fils d'ouvrier, l'un des fondateurs et aujourd'hui responsable, le plus élevé de Dzeta est conseiller d'une grande entreprise multinationale à laquelle il dit avoir emprunter un modèle de "maximalisation de l'efficacité » en le transférant sur l'ONG créée dans les années 2000 et enregistrée depuis peu. Son projet - dans lequel il a investi un capital initial - visait à diffuser dans les campagnes une idée de l'éducation comme un plaisir, un amusement, pour éviter la désertion des écoles jugées ennuyeuses et l'enrôlement des enfants dans le travail agricole. Dès 2005 Dzeta devient rentable, avec des subventions de grandes entreprises et de fondations chinoises et étrangères, les bénéfices de vente d'objets divers et les cotisations des volontaires. Petite-fille d'une famille d'artisans et de commerçants stigmatisés pour leur origine de classe « noire ", fille de " jeunes instruits » envoyés 10 ans à la campagne durant la révolution culturelle et travaillant à leur retour à Canton comme ouvriers, la directrice actuelle de Dzeta fut d'abord une volontaire enthousiaste de Dzeta, idéalisant la "pureté » et la « sincérité » de l'univers rural dans lequel elle s'était plongée étudiante lors d'un stage de l'organisation.

Depuis plus de 10 ans Dzeta envoie chaque année, après sélection sur dossier et entretien, puis formation d'une semaine dans la grande multinationale où travaille le responsable de Dzeta, durant les vacances scolaires des centaines d'étudiants de Canton volontaires dans des villages avec les autorités éducatives desquelles elle a institué une coopération. Les volontaires urbains sont en position professorale à l'égard des élèves ruraux et sont censés leur apprendre à apprendre, leur donner le goût de l'étude. Les documents filmés et écrits de Dzeta, les évaluations régulières de son action montrent un rapport hiérarchique archétypique entre citadins et campagnards, entretenu par des rites - tels les défilés avec le drapeau de Dzeta - et d'innombrables recommandations pour aborder ce qui se présente comme une réelle expédition. Dressons en la trame; du côté rural le manque absolu de tout est souligné: manque de nourriture, de loisirs, d'argent, de parents partis travailler en ville mais plus profondément représentation d'une scène de parenté détruite (par les abandons, les décès, les accidents, les divorces, etc.) qui fait des adolescents qu'on désigne comme les «élèves » des quasi-orphelins auxquels les jeunes étudiants vont de leur côté tout apporter dans un geste de charité magnanime. Dans les bagages éducatifs des citadins on trouve tout d'abord l'initiation et l'entrainement militaires - dont sont dépourvues les écoles rurales en regard des établissements scolaires urbains où elle est systématique - mais aussi des cours d'éducation physique, des jeux divers dont des jeux de rôle où chacun se raconte et au cours desquels les enfants de paysans pleurent beaucoup sur leurs malheurs, consolés par les étudiants de la ville qui donnent des cadeaux : stylos avec le sigle de Dzeta. « Développer l'esprit » des jeunes ruraux, par une " éducation participative », leur donner la possibilité "d'une meilleure vie » tout en devenant de « bons citoyens », « éradiquer les superstitions » telles sont les missions de « secours spirituel » de Dzeta, confiées aux étudiants volontaires qui doivent comprendre avec tact les «sentiments d'infériorité des paysans». Il s'agit d'une « responsabilité sociale » pour réparer « l'injustice » d'une éducation déficiente en milieu rural, opérée par des enseignants "mauvais », "bêtes». Des prix sont distribués aux «meilleurs élèves» et la cérémonie d'adieu - dans les larmes partagées - révèle la douleur immense d'une séparation citadins/paysans après une expérience d'intense communication fusionnelle. Les volontaires ont été prévenus auparavant que dans le canton « la poste ne marche pas, la police est corrompue par les guanxi et inefficace, 
l'hôpital est sale et on y soigne mal, Internet est lent » et que dans le village « il faut faire bouillir l'eau, ou mettre des capsules purificatrices, faire attention aux chiens et aux insectes, mais aussi aux vols et escroqueries, avoir emporté tous les médicaments avant de partir et aussi du thé, penser à la grippe, à la constipation due à la mauvaise nourriture, apporter du sucre, de l'huile camphrée pour les douleurs, etc. » C'est donc une véritable épreuve qui attend le courageux volontaire qui veut éduquer les enfants de paysans pendant ses vacances, donne à penser la documentation conseillée et le lecteur aura noté qu'on est ici très proche des visions humanitaires portées sur des terres de missions lointaines, africaines ou asiatiques en attente de la manne charitable de l'Occident. Un regard sur les investissements singuliers dont sont, de part et d'autre, l'objet des rencontres entre jeunes citadins et ruraux lors de ces stages de volontaires met en évidence l'enracinement du rapport hiérarchique mais aussi émotions contradictoires et prise de distance. Tournons-nous d'abord vers les volontaires urbains avec l'exemple éclatant de Talyan, membre du Parti depuis un an, fils d'une comptable et d'un ingénieur et petit-fils de deux lignées des classes supérieures. Ce grand jeune homme, doté d'une assurance parfaite, s'apprête à travailler comme programmateur télévisuel mais continue à consacrer toutes ses vacances au volontariat, entretenant des relations régulières et durables avec quelques adolescents et leurs familles dans plusieurs villages. Dans l'un de ces villages, il s'est rendu 8 fois en 2006, assistant aux fêtes, dormant dans les maisons. Talyan se perçoit comme « un grand frère » et il aime corriger ses « cadets » qui de son point de vue "parlent une langue grossière comme toujours à la campagne ». Aussi se félicite-t-il d'avoir fait pleurer une jeune fille « pour son bien » au point que le lendemain « elle a admis son erreur et ils sont devenus amis ». Il a pris le surnom de «petit cochon » pour être plus proche de tous ceux qu'il « éduque » et « force » à se tenir correctement en termes de politesse et de comportement. C'est à la campagne qu'il est tombé amoureux d'une autre volontaire, car explique-t-il il est impossible de penser épouser une femme inéduquée et dans les villages c'est très rare que les filles accèdent à l'éducation.

Dans la même perspective de domination condescendante une jeune volontaire cantonaise - fille unique d'un médecin et d'une fonctionnaire spécialisée dans le contrôle des naissances - se dit avoir été "très touchée sentimentalement " par son stage et s'avoue avoir été étonnée par l'imagination des élèves villageois en regard de ses conceptions antérieures "qu'à la campagne on n'a pas d'idées". C'est durant ce volontariat qu'elle a connu son futur époux. Le cas n'est pas rare et tout se passe comme si ces épisodes de "révélation" d'un autre monde - naturel et social avec lequel la communication reste très difficile faute de langue commune - encourageaient les unions entre citadins, se rapprochant dans le spectacle de l'abîme qui les sépare de l'autre.

Du côté des adolescents et de leurs familles qui reçoivent les volontaires urbains, la discordance est immédiate en regard de l'objectif de Dzeta. Une jeune fille, qui a trouvé à Canton, grâce à un réseau de parenté, un petit travail payé 800 yuans (logée, nourrie) dans un magasin de photocopie raconte ainsi : « Nos parents s'en fichent, mais nous nous pensions que les étudiants de la ville venaient s'amuser. J'avais 13 ans et je pensais à m'amuser avec eux. La première année c'était bien, j'ai participé, mais ils sont venus 3 ans de suite et c'était toujours pareil! Alors la deuxième année j'ai été deux jours et la troisième année, une seule journée ». Dans ce village, sur 40 élèves de sa classe, seuls 4 ou 5 aurait suivi - à l'en croire - les cours des volontaires, les autres devant travailler aux champs avec leurs parents. Quant à elle, elle dut affronter l'opposition de sa famille, jugeant que quatre trajets à vélo de 30 minutes par jour étaient « inutiles, simplement 
pour s'amuser ». Elle rentrait à midi déjeuner à la maison, faute de disposer du yuan unique nécessaire à l'achat d'un repas frugal à l'école. Elle insiste sur le fort sentiment d'infériorité ressenti face aux citadins, et sur le mépris et l'hostilité des enseignants locaux face à cette façon nouvelle d'apprendre.

En revanche il apparaît nettement que pour les jeunes filles qui souhaitent continuer leurs études et parviennent à aller à l'université à Canton, la présence des volontaires urbains les encourageant contre l'avis de leurs parents, privilégiant les garçons, a eu un poids important. Les jeunes citadins jouent alors le rôle d'un tiers étranger faisant pencher la balance du côté de l'étude, pour s'arracher à l'entre-soi et au travail agricole.

\section{Mutation et consolidation de l'Etat-parti}

La sociologie et l'anthropologie du bénévolat se sont principalement intéressées aux démocraties d'ancienne industrialisation, laissant de côté les pays cibles de l'action humanitaire, des ONG et de la charité occidentale au sens large. Cette concentration géographique intervient dans les orientations thématiques et les perspectives interprétatives: l'individualisation, l'autonomie comme norme individuelle, la production de soi, l'engagement autant associatif, politique que mural, sont appréhendés comme essentiels dans un cadre institutionnel et/ou collectif, perçu comme un étayage, ainsi que le développe en particulier Stephane Vermeersch ${ }^{3}$. Dans ce contexte, il va de soi que l'Etat est mis entre parenthèse, comme d'ailleurs le politique, puisque les acteurs évoluent dans une société où leur liberté est entière. Corollairement l'accent est mis, avec le paradigme maussien don/contre-don, sur le plaisir personnel retiré du bénévolat, voire la jouissance qu'il procure. Ces analyses, pour pertinentes qu'elles soient, manqueraient leur objectif si elles s'essayaient à être appliquées dans leur entièreté à d'autres configurations sociopolitiques, en particulier lorsque, comme en Chine, les acteurs sont sous la contrainte d'un régime autoritaire. Néanmoins - en dépit du fait que comme on l'a vu, l'auto-centrage du sujet "calmé ", " apaisé » par son investissement charitable, est présent dans les investigations menées - on ne saurait en déduire simplement que les processus d'individualisation sont empêchés ou inachevés en Chine. C'est la stratégie de l'Etat-parti qu'il faut ici remettre en scène en replaçant les organisations de volontaires dans un ensemble de dispositifs qui impliquent la charité et le souci des autres, care, sous différents volets : philanthropie d'entreprise, ONG encadrées, entreprises sociales, travail social. La mise en place d'une société de services, à laquelle tous ces agencements participent à leur manière, cristallise dans le même moment une mutation de la gouvernementalité de l'Etat-parti : celui-ci abandonne, en apparence, ses schèmes de verticalité implacable pour se donner une image bienveillante. Dans le même moment il s'agit d'entraîner les acteurs dans la gestion de la société, qu'ils s'engagent personnellement et aient le sentiment de remédier aux maux que l'Etat ne peut prendre en charge seul dans leur esprit. La charité est le maître-mot de cette nouvelle configuration au sein de laquelle les sujets pensent prendre des initiatives et les prennent effectivement, mais dans des structures où ils sont plus ou moins enrôlés comme les organisations de volontaires officielles, en voie d'enregistrement ou non encore enregistrées. Ces collectifs jouent sur l'émotion de chacun mais ne la libèrent qu'encadrée et orientée précisément. Tant qu'ils ne touchent pas à la défense des droits, ils sont encouragés par l'Etat et concourent à sa mutation et à sa pérennité, et ce d'autant plus qu'ils s'inscrivent dans un marché généralisé de services, adéquat à la croissance 
capitaliste initiée et poursuivie par le régime. La charité n'est alors pas seulement un tropisme subjectif et une onction sociale, elle se révèle un outil prédominant de gouvernance de l'Etat-parti qui rencontre là les régimes démocratiques, œuvrant avec eux pour l'efficience de cette nouvelle norme globale censée panser les plaies de l'hégémonie du marché et de la financiarisation de l'économie.

La charité fut dans le XIXe siècle européen une exemption dans l'imaginaire des rapports de domination et d'exploitation. Sa nature, dans un monde unifié par le capitalisme, estelle aujourd'hui totalement différente, si l'on prend en compte qu'elle s'est répandue dans toutes les couches sociales, neutralisant les structures de classe au profit de microhiérarchisations symboliques démultipliées et fluctuantes?

\section{NOTES}

1. En 20081 yuan $=1$ euro

2. Sorte de MSN chinois où les acteurs peuvent créer leur groupe de discussion.

3. « Ente individualisation et participation ", Revue française de sociologie, 2004/4 (Vol. 45)

\section{AUTEUR}

\section{MONIQUE SELIM}

UMR 201 IRD/PI. Développement et sociétés 